\title{
Radar Scattering from a Diffuse Vegetation Layer Over a Smooth Surface
}

\author{
NADER ENGHETA, STUDENT MEMBER, IEEE, AND CHARLES ELACHI, MEMBER, IEEE
}

\begin{abstract}
A simple model is presented for the oblique backscatter and bistatic scatter from a smooth surface overlain by a diffuse layer. Only single scattering in the diffuse layer is taken into account. The model analysis shows that the combination of volume scattering and oblique reflection at the surface may increase appreciably the waves scattering. The scattering strongly depends on the properties of the smooth surface. These results support some of the observations made with the Seasat spaceborne imaging radar over flooded regions with heavy vegetation cover.
\end{abstract}

\section{INTRODUCTION}

I N A RECENT PAPER, MacDonald et al. [5] reported that in their analysis of Seasat SAR data, they observed anomalous tonal variations in areas having relatively uniform and homogeneous vegetation canopy. They found that under certain conditions, the radar waves penetrate the vegetation canopy, and that high return is observed in region with standing surface water. Fig. 1 shows an example of a Seasat SAR image which illustrates this effect.

In this paper, we present a simple theoretical model which shows that the presence of a diffuse vegetation layer over a smooth surface could, in some cases, increase appreciably the backscattering cross section at oblique angles. In the absence of any vegetation layer, the backscattering cross section from a smooth surface is nul at oblique angles. If a layer of scatterer covers the smooth surface, the combination of scattering in the layer and reflection at the surface could lead to oblique backscatter larger than if the layer is by itself. Another way of viewing this effect was given by MacDonald et al. [5]. As the wave penetrates the layer, its amplitude and phase are modified randomly. When this wave interacts with the smooth surface, the effect is similar to the case of the plane wave with a randomly rough surface.

The problem of scattering from a scattering layer using the Dyson equation and the first-order normalization method was investigated by Fung [3]. In this paper, we present a very simple model which seems to explain the effect observed experimentally and gives an approximate solution for the backscattering of linear and circular waves and the bistatic scattering of linear waves. It is assumed that the vegetation layer

Manuscript received April 14, 1981; revised September 22,1981. This paper presents the results of one phase of research carried out at the Jet Propulsion Laboratory, California Institute of Technology, under Contract NAS7-100, sponsored by the National Aeronautics and Space Administration.

N. Engheta is with the California Institute of Technology, Pasadena, CA 91125 .

C. Elachi is with the Jet Propulsion Laboratory, California Institute of Technology, Pasadena, CA 91109.

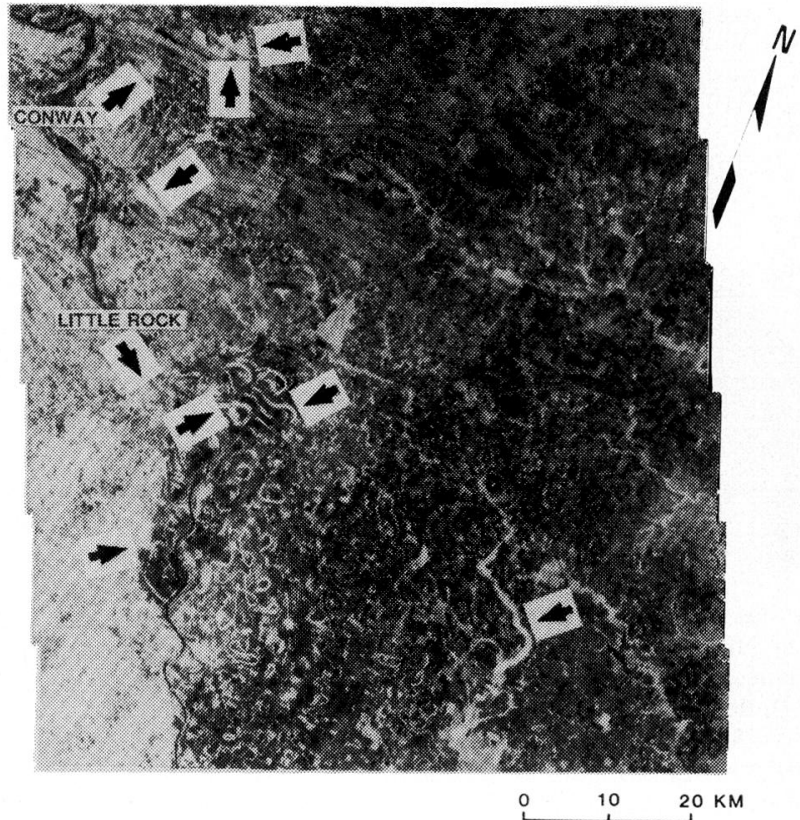

Fig. 1. Seasat radar image of the east-central area in Arkansas acquired on July 24, 1978. Arrows indicate anomalous radar returns. They all correspond to areas of standing water covered partially or fully by a vegetation canopy. The three arrows in the upper left of the image (near Conway) indicate large swamp areas with a discontinuous deciduous forest canopy (about 85 percent cover). Most of the other arrows indicate axbow lakes or abandoned meanders along the Arkansas river (courtesy of H. MacDonald).

cover be represented by a uniform layer of particles small relative to the wavelength. In the case of the Seasat SAR, the radar wavelength was $24 \mathrm{~cm}$.

In Section II, we present the general theoretical formulation for the backscatter with linear polarization. In Section III, we discuss the special case of small scattering particles. In Sections IV and $\mathrm{V}$, we consider the case of circular polarization. Finally, in Section VI, we discuss the case of bistatic scattering. In all our analysis we neglect multiple scattering in the diffuse layer.

\section{Formulation of the Backscattering Cross Section for Linear Polarization}

There are four possible combinations of single scattering and reflection which can lead to a backward return of the incident wave. These four cases are illustrated in Fig. 2. The backscattering cross sections for the four cases are given by

$$
\sigma_{1}=\sigma(\theta, \theta) e^{-\alpha_{1}}
$$




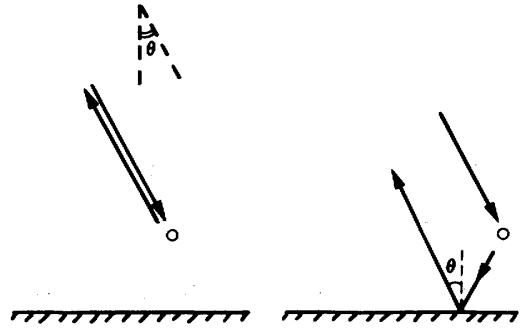

(a) (b)

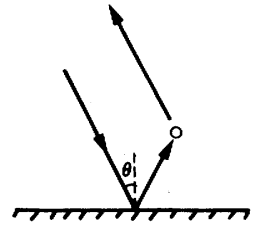

(c)

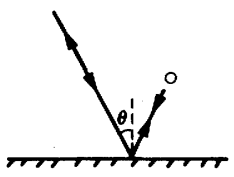

(d)

Fig. 2. Different ray tracing configurations which contribute to the backscatter signal as a result of reflection at the interface and/or scattering from the particles in the diffuse layer.

$$
\begin{aligned}
& \sigma_{2}=R \sigma(\theta, \Pi-\theta) e^{-\left(\alpha_{1}+\alpha_{2}\right)} \\
& \sigma_{3}=R \sigma(\Pi-\theta, \theta) e^{-\left(\alpha_{1}+\alpha_{2}\right)}=\sigma_{2} \\
& \sigma_{4}=R^{2} \sigma(\Pi-\theta, \Pi-\theta) e^{-\left(\alpha_{1}+2 \alpha_{2}\right)}
\end{aligned}
$$

where

$$
\begin{aligned}
& R \quad \begin{array}{l}
\text { Fresnel power reflection coefficient at the surface } \\
\text { with an incidence angle equal to } \theta ;
\end{array} \\
& \sigma(\phi, \gamma) \quad \begin{array}{l}
\text { bistatic scattering cross section with incoming angle } \\
\phi \text { and scattering angle } \gamma ;
\end{array} \\
& \alpha_{1} \quad \begin{array}{l}
\text { round-trip loss in the layer above the scattering } \\
\text { element; } \\
\alpha_{2} \quad \begin{array}{l}
\text { round-trip loss in the layer between the scattering } \\
\text { element and the surface. }
\end{array}
\end{array} \\
&
\end{aligned}
$$

The backscattering cross section for a typical particle is

$$
\begin{aligned}
\sum_{t}= & \sum_{n=1}^{4} \sigma_{n}=\left[\sigma(\theta, \theta)+2 R \sigma(\theta, \Pi-\theta) e^{-\alpha_{2}}\right. \\
& \left.+R^{2} \sigma(\Pi-\theta, \Pi-\theta) e^{-2 \alpha_{2}}\right] e^{-\alpha_{1}} .
\end{aligned}
$$

Because the scattering element has equal probability to be at any location in the layer, then by integrating over the thickness of the scattering layer we get

$$
\begin{aligned}
\sum_{t}= & \frac{e^{-\alpha}}{\alpha}\left[\sigma(\theta, \theta)+2 R \sigma(\theta, \Pi-\theta) \frac{\alpha e^{-\alpha}}{\sinh \alpha}\right. \\
& \left.+R^{2} \sigma(\Pi-\theta, \Pi-\theta) e^{-2 \alpha}\right] \sinh \alpha
\end{aligned}
$$

where $\alpha=\gamma L / \cos \theta, \gamma$ is the loss per unit length, and $L$ is the layer thickness.

\section{Special Case of Small Spherical Particles}

The most simple case is when the scattering particles size is much smaller than the wavelength (i.e., $\lambda>4 \Pi a, a=$ particles size). In this case, the scattering is given by [1]

$$
\sigma(\Pi-\theta, \Pi-\theta)=\sigma(\theta, \theta)=\sigma_{0}=4 \Pi a^{2}\left|\frac{\epsilon_{p}-1}{\epsilon_{p}+2}\right|^{2}\left(k_{0} a\right)^{4}
$$

and

$$
\sigma(\theta, \Pi-\theta)=4 \Pi a^{2}\left|\frac{\epsilon_{p}-1}{\epsilon_{p}+2}\right|^{2}\left(k_{0} a\right)^{4}
$$

when the electric vector is perpendicular to the plane of inci- dence, i.e., horizontal polarization, and

$$
\sigma(\theta, \Pi-\theta)=4 \Pi a^{2}\left|\frac{\epsilon_{p}-1}{\epsilon_{p}+2}\right|^{2}\left(k_{0} a\right)^{4} \cos ^{2}
$$

when the electric vector is in the plane of incidence, i.e., vertical polarization. In the above expressions, $\epsilon_{p}$ is the relative dielectric constant of the particles and $k_{0}=2 \Pi / \lambda$.

The backscattering cross section is then given by [from (2)]

$$
\sum_{t}=\sum_{H H}=\sigma_{0} \frac{e^{-\alpha}}{\alpha} \sinh \alpha\left[1+2 R_{\perp} \frac{\alpha e^{-\alpha}}{\sinh \alpha}+R_{\perp}^{2} e^{-2 \alpha}\right]
$$

when the electric vector is perpendicular to the plane of incidence, and

$$
\sum_{t}=\sum_{V V}=\frac{\sigma_{0} e^{-\alpha}}{\alpha} \sinh \alpha\left[1+2 R_{\|} \cos ^{2} 2 \theta \frac{\alpha e^{-\alpha}}{\sinh \alpha}+R_{\|}^{2} e^{-2 \alpha}\right]
$$

when the electric vector is in the plane of incidence. In the above expressions, $R$ is the Fresnel power reflection coefficient which is given by Jordan and Balmain [4]. The loss coefficient $\gamma$ is equal to the absorption loss (the scattering loss is negligible when $\lambda \gg a$ ) in $\mathrm{m}^{-1}$ and is given by [2]

$$
\gamma=\frac{1.8 \Pi}{\lambda \rho} 10^{-3} \frac{\epsilon_{p 2} M}{\left(\epsilon_{p 1}+2\right)^{2}+\epsilon_{p 2}^{2}}
$$

where $\epsilon_{p_{1}}, \epsilon_{p_{2}}$ are the real and imaginary part of the particles relative dielectric constant, $M$ is the mass density of the scattering layer in $\mathrm{g} / \mathrm{m}^{3}, \rho$ is the density of the particles in $\mathrm{g} / \mathrm{cm}^{3}$, and $\lambda$ is the wavelength in $\mathrm{cm}$.

The behavior of $A=\Sigma_{t} \alpha / \sigma_{0} e^{-\alpha} \sinh \alpha$ as a function of the observation geometry and the relative dielectric constant $\epsilon$ is illustrated in Fig. 3. $A$ represents the additional factor which results from the presence of a reflecting surface below the scattering layer.

In the case of a thick layer, $\gamma L \gg 1$ and $A=1$, the presence of the reflecting surface has no effect. This is due to the fact that almost no energy penetrates through the layer and get reflected by the surface.

Another special case is when $\gamma L=0$ (i.e., low density scattering layer, negligible absorption). Then

$$
\begin{aligned}
& A_{H H}=\left(1+R_{\perp}\right)^{2} \\
& A_{V V}=1+2 R_{\|} \cos ^{2} 2 \theta+R_{\|}^{2} .
\end{aligned}
$$



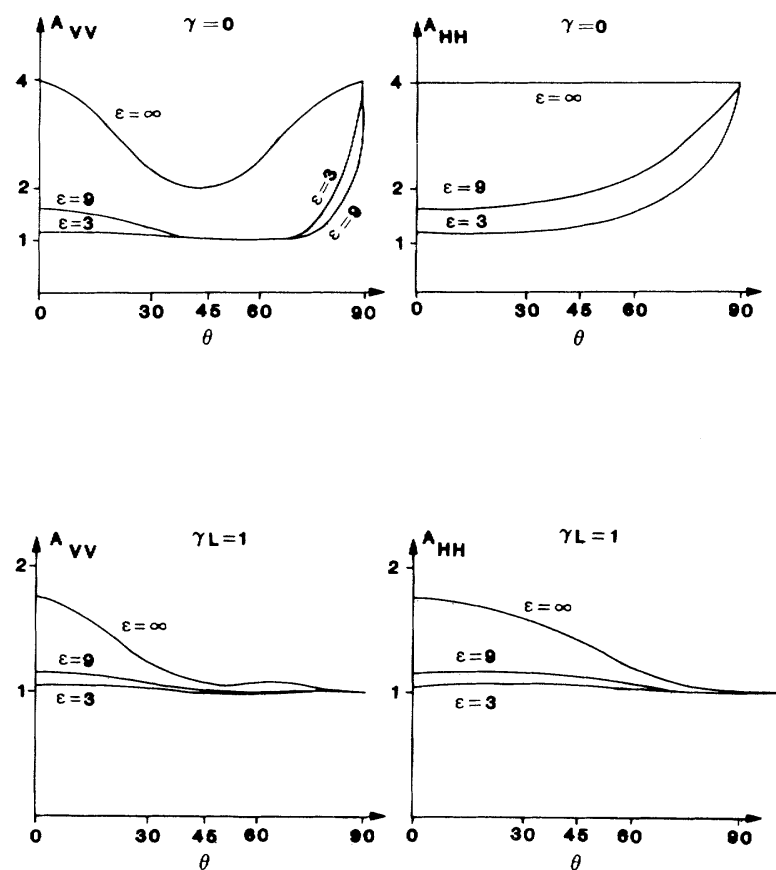

Fig. 3. Plots of the additional factors $A_{H H}$ and $A_{V V}$ for different values of $\alpha$ (losses in the diffuse layer) and $\epsilon$ (dielectric constant of the flat interface). These plots correspond to the case of linear polarization.

In the limit of a perfectly reflecting surface (such as a water layer) then

$$
\begin{aligned}
& A_{H H}=4 \\
& A_{V V}=2\left(1+\cos ^{2} 2 \theta\right) .
\end{aligned}
$$

Thus the backscattering cross section is increased by 3 to $6 \mathrm{~dB}$ due to the presence of the totally reflecting surface.

Fig. 3 shows $A_{V V}$ and $A_{H H}$ as a function of the incidence angle. We considered the cases of $\epsilon=3,9$, and $\infty$ (perfectly reflecting surface) and $\gamma L=0$ and 1 . It is clear that for small $\gamma L$ (i.e., appreciable penetration in the vegetation layer), the surface interface can play an appreciable role. For instance, in the case of the Seasat radar configuration $\left(H H, 20^{\circ}\right.$ incidence angle), the change in the surface dielectric constant from $\epsilon=3$ to $\epsilon=\infty$ leads to an increase in the backscatter by $4 / 1.2=3.3$ (i.e., $5.2 \mathrm{~dB}$ ). This is qualitatively consistant with the Seasat SAR observations over swampy and flooded regions with vegetation cover (Fig. 1).

\section{Formulation of the Backscattering Cross Section for Circular Polarization}

The four backscatter configurations are the same as in Fig. 2. For the configuration where only scattering occurs (Fig. 2(a)), we have (neglecting absorption loss for the time being)

$$
\begin{aligned}
& \sigma_{R L}=\sigma_{L R}=\sigma_{0} \\
& \sigma_{L L}=\sigma_{R R}=0
\end{aligned}
$$

where the subscript $L$ and $R$ refers to right and left circular polarization.

Let us consider next the configuration in Fig. 2(b) (i.e., scattering and reflection). The incident wave is given by (see

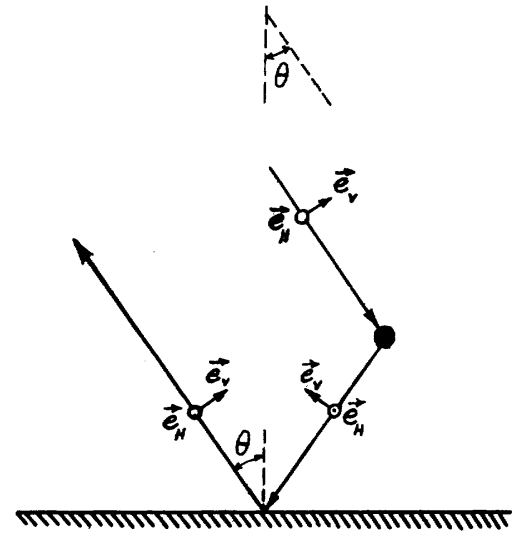

Fig. 4. Geometry for circularly polarized wave.

Fig. 4)

$$
\boldsymbol{E}_{\boldsymbol{i}}=\boldsymbol{E}_{v} \pm i \boldsymbol{E}_{H}
$$

where \pm corresponds to the right and left circular polarization, respectively. The scattered field from the particle is equal to

$$
\boldsymbol{E}_{S}=S_{1}(\theta) E_{v} \boldsymbol{e}_{v} \pm i S_{2}(\theta) E_{H} \boldsymbol{e}_{H}
$$

and after reflection

$$
\boldsymbol{E}_{\boldsymbol{r}}=S_{1}(\theta) R_{\|}^{\prime} E_{v} \boldsymbol{e}_{v} \pm i S_{2}(\theta) R_{\perp}^{\prime} E_{H} \boldsymbol{e}_{H}
$$

where the prime corresponds to the amplitude reflection coefficient $\left(R=R^{\prime 2}\right)$. For a circularly polarized incident wave $E_{H}=E_{v}=E_{0}$. Then

$$
E_{r}=\left(S_{1}(\theta) R_{\|}^{\prime} e_{v} \pm i S_{2}(\theta) R_{\perp}^{\prime} e_{H}\right) E_{0}
$$

which is an elliptically polarized return. An elliptically polarized wave can be subdivided into two circularly polarized waves. Therefore, we get

$$
\begin{gathered}
\sigma_{R R}=\sigma_{L L}=\left|\frac{S_{1}(\theta) R_{\|}^{\prime}-S_{2}(\theta) R_{\perp}^{\prime}}{2}\right|^{2} \\
\sigma_{R L}=\sigma_{L R}=\left|\frac{S_{1}(\theta) R_{\|}^{\prime}+S_{2}(\theta) R_{\perp}^{\prime}}{2}\right|^{2} .
\end{gathered}
$$

The same expressions apply to the configuration in Fig. 2(c).

In the case of scattering and double reflection (Fig. 2(d)), following the same steps as above and remembering that the scattering at the particle is backward not bistatic, then we find that

$$
\begin{gathered}
\sigma_{R R}=\sigma_{L L}=\sigma_{0}\left|\frac{R_{\perp}-R_{\|}}{2}\right|^{2} \\
\sigma_{R L}=\sigma_{L R}=\sigma_{0}\left|\frac{R_{\perp}+R_{\|}}{2}\right|^{2} .
\end{gathered}
$$

Therefore, the total backscattering cross section is given by

$$
\begin{aligned}
\sum_{R R}=\sum_{L L}= & {\left[2\left|\frac{S_{1}(\theta) R_{\|}^{\prime}-S_{2}(\theta) R_{\perp}^{\prime}}{2}\right|^{2} e^{-\alpha}\right.} \\
& \left.+\sigma_{0}\left|\frac{R_{\perp}-R_{\|}}{2}\right|^{2} \frac{\sinh \alpha}{\alpha} e^{-2 \alpha}\right] e^{-\alpha}
\end{aligned}
$$



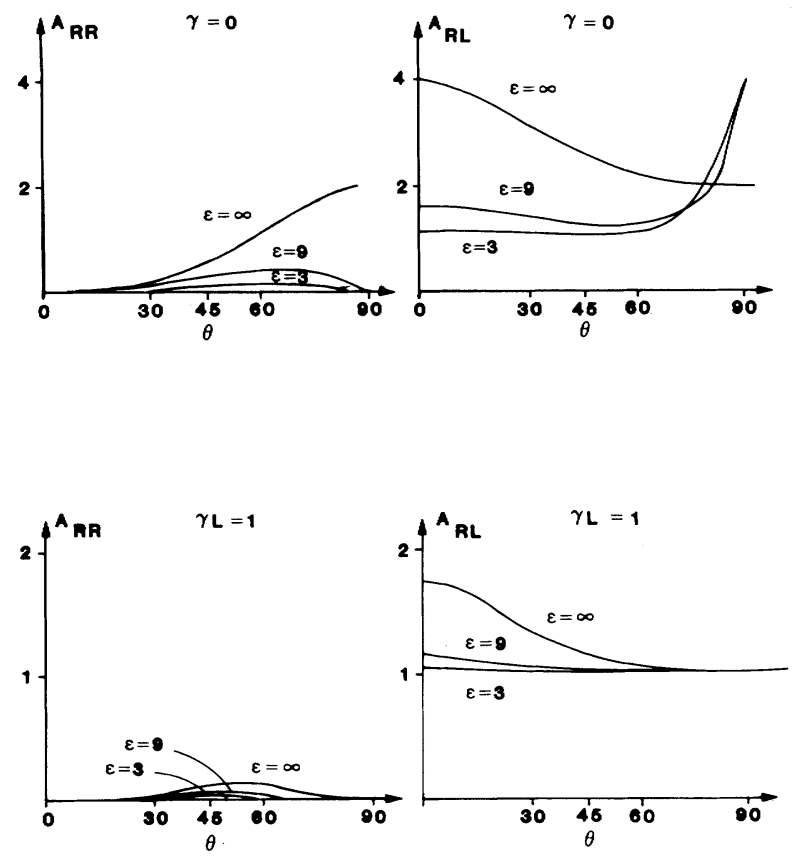

Fig. 5. Plots of $A_{R R}$ and $A_{R L}$ for the different values of $\alpha$ and $\epsilon$. These plots correspond to the case of circular polarization.

$$
\begin{aligned}
\sum_{R L}=\sum_{L R}= & {\left[\sigma_{0} \frac{\sinh \alpha}{\alpha}+2\left|\frac{S_{1}(\theta) R_{\|}^{\prime}+S_{2}(\theta) R_{\perp}^{\prime}}{2}\right|^{2} e^{-\alpha}\right.} \\
& \left.+\sigma_{0}\left|\frac{R_{\perp}+R_{\|}}{2}\right|^{2} \frac{\sinh \alpha}{\alpha} e^{-2 \alpha}\right] e^{-\alpha}
\end{aligned}
$$

\section{Case of Small Spherical Particles}

In the case of small spherical particles we have

$$
\begin{aligned}
& S_{1}(\theta)=-\sqrt{\sigma_{0}} \cos 2 \theta \\
& S_{2}(\theta)=\sqrt{\sigma_{0}}
\end{aligned}
$$

and the backscattering cross section is equal to

$$
\begin{aligned}
\sum_{R R}=\sum_{L L}= & \sigma_{0} e^{-2 \alpha}\left[\frac{\left|R_{\|}^{\prime} \cos 2 \theta+R_{\perp}^{\prime}\right|^{2}}{2}\right. \\
& \left.+\frac{\sinh \alpha}{\alpha}\left|\frac{R_{\|}-R_{\perp}}{2}\right|^{2} e^{-\alpha}\right] \\
\sum_{R L}=\sum_{L R}= & \sigma_{0} e^{-\alpha} \frac{\sinh \alpha}{\alpha}\left[1+\frac{\left|R_{\|}^{\prime} \cos 2 \theta-R_{\perp}^{\prime}\right|^{2}}{2} \frac{\alpha}{\sinh \alpha} e^{-\alpha}\right. \\
& \left.+\left|\frac{R_{\|}+R_{\perp}}{2}\right|^{2} e^{-2 \alpha}\right] .
\end{aligned}
$$

In Fig. 5, we plotted $A_{R R}=A_{L L}=\Sigma_{R R} \alpha / \sigma_{0} \sinh \alpha e^{-\alpha}$ and $A_{R L}=A_{L R}=\Sigma_{R L} \alpha / \sigma_{0} \sinh \alpha e^{-\alpha}$ for different cases. In the case of direct polarized return (i.e., $\Sigma_{R L}$ and $\Sigma_{L R}$ ), $A$ represents the additional factor which results from the presence of the reflecting surface. In the case of depolarized return (i.e., $\Sigma_{R R}$ and $\Sigma_{L L}$ ), $A$ represents the total factor relative to the polarized return without the reflecting surface.

One special case is when $\gamma L \gg 1$. Then

$$
A_{R L}=A_{L R}=1
$$

$$
A_{R R}=A_{L L}=0
$$

as expected.

Another special case is when $\gamma L=0$ and $R_{\|}=R_{\perp}=1$, $\left(R_{\perp}^{\prime}=-1, R_{\|}^{\prime}=1\right)$. Then

$$
\begin{aligned}
& A_{R L}=A_{L R}=2+\frac{(1+\cos 2 \theta)^{2}}{2} \\
& A_{L L}=A_{R R}=\frac{(1-\cos 2 \theta)^{2}}{2} .
\end{aligned}
$$

For the case of large $\epsilon$, the direct polarization factor $\left(A_{R L}=\right.$ $A_{L R}$ ) varies from 4 at normal incidence to 2 at grazing incidence. The depolarized factor varies from zero at normal incidence to 2 at grazing incidence.

\section{Bistatic Scattering}

The approach used in the previous sections to derive the backscattering cross section can also be used to derive the bistatic scattering cross section. Assuming that the incoming and outgoing waves are in the same plane, and that only single scattering is accounted for, the different ray tracing possibilities are shown in Fig. 6. For scattering particles that are nondirectional, the scattering cross section for the four configurations in Fig. 6 are given by, respectively,

$$
\begin{aligned}
& \sigma_{1}=\sigma(\theta, \phi) \\
& \sigma_{2}=\sigma(\theta, \Pi-\phi) R(\phi) \\
& \sigma_{3}=\sigma(\Pi-\theta, \phi) R(\theta) \\
& \sigma_{4}=\sigma(\Pi-\theta, \Pi-\phi) R(\theta) R(\phi) .
\end{aligned}
$$

The scattering cross section is then equal to

$$
\sigma_{t}=\sigma(\theta, \phi)[1+R(\theta) R(\phi)]+\sigma(\theta, \Pi-\phi)[R(\theta)+R(\phi)]
$$

where the effect of losses in the diffuse layer has been neglected and the particles are assumed to be symmetric. The additional factor due to the interface is then

$$
\begin{aligned}
A & =\frac{\sigma_{t}}{\sigma(\theta, \phi)} \\
& =1+R(\theta) R(\phi)+[R(\theta)+R(\phi)] \sigma(\theta, \Pi-\phi) / \sigma(\theta, \phi) .
\end{aligned}
$$

This shows that the interface does increase the bistatic cross section. It can be very large if $\sigma(\theta, \Pi-\phi)$ is much larger than $\sigma(\theta, \phi)$. Using the data given by Barrick [2, fig. 3-7], we give the following examples to illustrate this point. For $a=3 \lambda / \Pi$ then

$$
\frac{\sigma(0,150)}{\sigma(0,30)} \simeq 250
$$

for vertical polarization, and

$$
\frac{\sigma(25,155)}{\sigma(25,25)}=3500
$$

for horizontal polarization.

In the case of isotropic scattering by the particles, $A$ becomes $A=[1+R(\theta)][1+R(\phi)]$. 


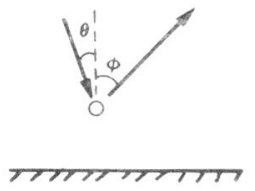

(a)

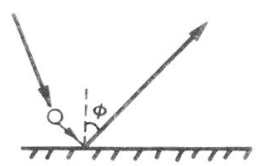

(b)

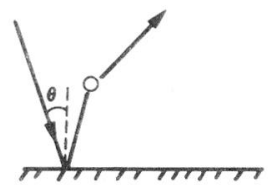

(c)

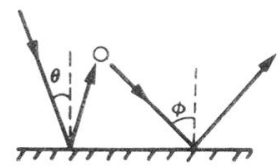

(d)

Fig. 6. Different ray tracing configurations which contribute to the scattered signal (bistatic) as a result of reflection at the interface and/ or scattering from the particles in the diffuse layer.

\section{CONCLUSION}

If the electromagnetic energy is not appreciably absorbed or scattered by the diffuse layer, the bottom interface will play an important role in oblique backscattering even if it is perfectly smooth. This effect is also important in bistatic scattering. In the case of the Seasat radar data shown in Fig. 1, the theory presented in this paper verifies the observation by MacDonald et al. [5] that the presence of a water interface (due to flooding) below the vegetation canopy will increase the surface scattering at oblique angles. We also presented how this effect depends on the polarization of the wave and its incidence angle.

The theory presented here is based on simple ray tracing and single scattering. In a future paper, we will present the case of multiple scattering and a rough interface surface.

\section{REFERENCES}

[1] D. E. Barrick, Radar Cross Section Handbook, vol. 1, G. T. Ruck, Ed. New York: Plenum Press, 1970, ch. 3.

[2] L. J. Battan, Radar Observation of the Atmosphere. Chicago, IL: Univ. of Chicago Press, 1973.

[3] A. K. Fung, "Scattering from a vegetation layer," IEEE Trans. Geosci. Electron., vol. GE-17, pp. 1-6, 1979.

[4] E. C. Jordan and K. G. Balmain, Electromagnetic Waves and Radiating Systems. Englewood Cliffs, NJ: Prentice Hall, 1968.

[5] H. C. MacDonald, W. P. Waite, and J. S. Demarcke, "Use of Seasat satellite radar imagery for the detection of standing water beneath forest vegetation," presented at the Amer. Soc. of Photogrammetry, Ann. Tech. Meet., Niagara Falls, NY, 1980.

Nader Engheta (S'80) was born in Tehran, Iran, on October 8, 1955. $\mathrm{He}$ received the B.S. degree (honors) in electrical engineering from the University of Tehran in 1978. He entered the California Institute of Technology in Septernber 1978 and received the M.S. degree in electrical engineering in June 1979.

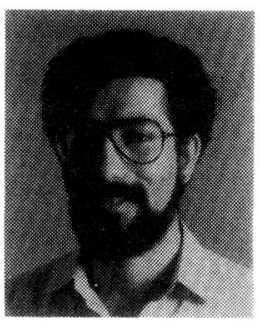

At present he is working towards the Ph.D. degree at the California Institute of Technology in electromagnetic theory and wave propagation. He has been a Research Assistant at the California Institute of Technology, Pasadena, since 1979. He has also been part time associated with the Jet Propulsion Laboratory, since July 1980, investigating theoretical problems related to remote sensing.

Mr. Engheta is a member of APS

Charles Elachi (M'71) was born in Rayak, Lebanon, on April 18, 1947. He received the "Ingenieur" degree in radioelectricity with honors and the "Prix de Houille Blanche" from the Polytechnic Institute of Grenoble, Grenoble, France, in 1968, and the B.S. degree in physics from the University of Grenoble in 1968. He received the M.S. and Ph.D. degrees in electrical engineering and business economics from the California Institute of Technology, Pasadena, in 1969 and 1971, respectively.

He has worked at the Physical Spectrometry Laboratory, University of Grenoble, on plasma in microwave cavities. He was a Teaching Assistant at Caltech in 1969. In 1970 he joined the Space Sciences Division, Jet Propulsion Laboratory, Pasadena, where he is presently Leader of the Radar Remote Sensing Team, which is involved in investigating spacecraft-borne scientific experiments for planetary and Earth studies using coherent radar techniques. Since 1980, he has been the Manager for Radar Development which covers all aspects of the radar remote sensing program at JPL. He is the principal investigator on the Shuttle Imaging Radar. He is also involved in studying theoretical electromagnetic problems related to scattering from natural terrain, remote sensing, stratified media, space-time periodic media, and DFB lasers. He has 130 papers, patents, reports, and conference presentations in the above fields.

In 1973, Dr. Elachi was the first recipient of the R. W. P. King award. In 1980, he received the Autometric Award of the American Photogrammetric Society. He is a member of AAAS and Sigma Xi. 